\title{
Non-operative Management of Anterior Knee Pain: Patient Education
}

\author{
Lauren Bosshardt ${ }^{1} \cdot$ Taylor Ray $^{1} \cdot$ Seth Sherman ${ }^{1,2}$ (i) \\ Accepted: 17 December 2020 / Published online: 1 February 2021 \\ (C) The Author(s), under exclusive licence to Springer Science+Business Media, LLC part of Springer Nature 2021
}

\begin{abstract}
Purpose of Review Anterior knee pain is a multifactorial, often chronic condition, which can lead to long-term pain and disability. The purpose of this review is to examine the latest research on patient education for anterior knee pain. We will focus on how we might use patient education to increase the adoption of other efficacious treatment modalities and offer some suggestions for content and form of effective patient education.

Recent Findings New research suggests that patient education, alone, or in combination with targeted exercise therapy, can be effective in reducing pain and improving function in patients with patellofemoral pain. Addressing non-physical or psychological factors may also be an important component of patient education in many patients with chronic pain. Incorporation of new technologies into patient education, such as those available online, or through phone- or tablet-based apps, is likely to be helpful in the future, as we move more towards connecting with patients virtually.

Summary Patient education has been shown to be effective in decreasing pain and improving activity in patients with patellofemoral pain. Patient education should be individualized to the patient, focus on the latest effective treatments, and emphasize those treatments that can be self-managed by the patient. Emphasis should also be placed on patient understanding of risk factors and patterns of movement that may lead to, or exacerbate, anterior knee pain. Future research should continue to further characterize the elements of patient education that offer the most efficient treatment benefit.
\end{abstract}

Keywords Patellofemoral pain · Anterior knee pain · Patient education · Pain management · Physical therapy modalities

\section{Introduction}

The most common cause of anterior knee pain is diagnosed as patellofemoral pain and is characterized by vague and diffuse anterior knee pain, located around or behind the patella,

This article is part of the Topical Collection on Non-Operative Management of Anterior Knee Pain

Seth Sherman

shermans@stanford.edu

Lauren Bosshardt

laurenbosshardt@gmail.com

Taylor Ray

raytay@stanford.edu

1 Department of Orthopedic Surgery, Stanford University, Stanford, CA, USA

2 Stanford Medicine Outpatient Center, 450 Broadway, Pavilion A, Redwood City, CA 94063, USA during loading of the patellofemoral joint with weight bearing activity [1]. The annual incidence of patellofemoral pain has been reported as high as $23 \%$ in adults and $28 \%$ in adolescents [2]. Current evidence-based treatment options include exercise therapy, with or without other interventions (taping or bracing, foot orthoses, or manual therapy) [3]. However, despite these treatment options, roughly $50 \%$ of people may experience persistent patellofemoral pain [4], some of them having persistent symptoms for many years [5].

These reported poor long-term outcomes indicate that there is a need for additional treatment options and self-guided techniques that can be continued longer term. One strategy to try to improve longer term outcomes is to provide dedicated patient education: patient-specific advice on suspected etiology(s), proposed options for treatment, and expectation management. We hypothesize that patient education, alone or in combination with exercise therapy, can be effective in reducing pain and improving function in patients with patellofemoral pain. Unfortunately, most published literature on patient education does not clearly characterize the form or 
content of this education. Despite this limitation, the purpose of this article will be to summarize the recent available literature related to patient education for anterior knee pain, as well as to compile a collection of valuable resources of various forms of patient education.

\section{Effectiveness of Patient Education}

A recent systematic review found evidence to suggest that patient education delivered by a healthcare provider may result in similar decreases in pain and increases in function, when compared to patient education combined with directed exercise therapy, taping, or gait retraining interventions. In three of the included trials, education was delivered in the form of leaflets or booklets, and in five trials, education was delivered via a health professional. Efficacy was evaluated by comparing pain and function scores after intervention(s). However, there is no detailed information regarding the specific content of education delivered verbally by a health professional, or about the amount of time spent in education [6••].

Recent research looking at online education programs for other musculoskeletal conditions have been effective in decreasing pain and improving pain-coping skills in patients with chronic knee pain [7], and hip osteoarthritis [8]. In a more direct comparison, education compared to no treatment in knee osteoarthritis has been shown to decrease pain and improve quality of life and function [9], suggesting that this approach may be effective in other musculoskeletal conditions like patellofemoral pain.

\section{Content of Education}

The particular content of patient education was often not specified in research studies looking into the effectiveness of patient education. However, broad categories have been recommended, including the following: (i) explanation on the nature and possible causes of PFP; (ii) advice on load management, and (iii) advice on self-management of pain [6••].

Patient education can also take a variety of forms, including verbal/in person, written (handout, pamphlet, article, or book), or online (including written, video, and app-based). As previously mentioned, the most effective content and form will vary depending on individual patient characteristics.

Patient education needs to be tailored to the individual, so the first step in providing appropriate patient education is to seek to understand your patient. Most physicians do this intuitively and are able to form an understanding of the patient, identifying risk factors through careful history taking and exam. The form and content of patient education will also need to be adjusted for the availability of resources (does the patient have internet access?), as well as educational and reading level. Assessing the patient's current insight into their disease process and his or her individual goals of care is also important in the development of individualized patient education.

\section{Explanation of Possible Causes}

Despite being very common, the exact pathophysiology of patellofemoral syndrome is not well understood and is likely the result of a complicated interplay of biomechanical, behavioral, and psychological components. For this reason, we should acknowledge the challenges in trying to provide patient education for a syndrome with such varied contributions and targeted treatments.

Although we often call patellofemoral pain a "syndrome," it lacks the specific combination of symptoms, signs, imaging findings, and/or serum values that we typically use to more precisely diagnose other "syndromes" in medicine, and this has led to some controversy in the terminology we use to describe patellofemoral pain, with some arguing that we should instead stick to a more general description of "anterior knee pain" [10]. However, there may be some value, in terms of patient education, in giving the disease a name.

According to a recent review highlighting recent updates in risk factors and treatment of patellofemoral pain, risk factors are varied and include biomechanical stress, hip weakness, quadriceps weakness, VMO delayed activation, foot overpronation, flexibility (patellar hypermobility or maltracking), or inflexibility. Each of these risk factors has a proposed targeted treatment [11]. These findings are summarized in Table 1. Helping the patient understand underlying personal risk factors provides a better framework for understanding how to correct those underlying risk factors, which may be multiple and cumulative. Again, this is why it seems appropriate to target patient education to the identified patientspecific problems.

An example of a good way to explain possible causes might be to focus on one or two most likely contributing factors for a particular individual. Patients may be better able to understand and focus on one or two factors that are most likely contributing to their particular case, rather than get lost in the interconnected web of a myriad of lesser contributors.

\section{Advice on Load Management}

Training errors and overuse may play a large role in the development of patellofemoral pain, so correcting these errors through patient education could be particularly effective. One theory of pathophysiology suggests that patellofemoral syndrome is a result of the loss of homeostasis. According to this theory, pain results from overloading bone and synovial tissues underlying the patellofemoral joint. This could be from a 
Table 1 Nature and causes of patellofemoral pain

\begin{tabular}{ll}
\hline Risk factor/cause & Treatment recommendations \\
\hline Hip muscle/hip abductor weakness & Hip strengthening \\
Quadriceps weakness & Quadriceps strengthening, blood flow restriction \\
Delayed VMO activation & Biofeedback \\
Patellar maltracking or hypermobility & Patellar taping or bracing \\
Foot overpronation & Core foot strengthening and foot orthosis \\
Soft tissue inflexibility & Stretching, manual soft tissue therapy \\
Overtraining & Load management, return to activity plan \\
Gait deviations & Gait retraining \\
Non-physical-fear avoidance and & Address beliefs towards pain, unmet expectations, patient \\
catastrophizing & engagement \\
\hline
\end{tabular}

multitude of external forces that increase stress across the patellofemoral joint, including overuse, improper running biomechanics, excessive weight, or unsuitable footwear [12].

Return to play or return to activity guidelines may be particularly helpful to avoid reinjury or the development of a new injury. A study in distance runners found that increasing mileage by greater than $30 \%$ over 2 weeks made them much more likely to get injured [13]. This rough estimation can be utilized to help design a training program that will not increase the training load above the patient's load capacity.

An example of a good way to advise on load management might be to quickly go through or ask about the most common pitfalls in training during the initial evaluation. This not only helps the healthcare professional identify these training errors but also highlights these as areas of attention for the patient. Specifically targeting training for running with a sort of "running intake questionnaire" may be especially helpful to identify common training errors in typical run progression, useful equipment (i.e., footwear modification), and warning signs for overuse injury. Having the patient try to quantify the load of the past few weeks of training (in terms of both volume and intensity) or reviewing a training log, if available, can be helpful in highlighting specific pitfalls in the individual's training plan.

\section{Self-Management of Pain}

An essential component of patient education is how to self-manage pain. Patients who are empowered to control and treat their own symptoms develop a sense of self-efficacy.

Self-efficacy is an integral part of self-management and refers to the general mindset of confidence that a patient might feel in being able to exert control and manage one's own motivation, behavior and future obstacles and challenges [14]. Online programs that have included patient instruction in self-efficacy have been effective in reducing pain and improving quality of life and function scores in patients with knee osteoarthritis [9].

Similarly, in a study examining self-management in patients with patellofemoral pain, the "locus of control" (closely related to the psychological construct of selfefficacy) emerged as a key facilitator in self-managed exercises and return to physical activity. Patients that felt an internal locus of control were better able to appreciate when they were approaching their clinical and personal goals [15•]. Table 2 contains some basic suggestions for categories of self-management. More specific tools for self-management can be found in Table 3 .
Table 2 Summary of selfmanagement strategies

\begin{tabular}{ll}
\hline Self-management & Explanation \\
\hline $\begin{array}{l}\text { Athletic/walking shoes } \\
\text { Kinesiology tape }\end{array}$ & $\begin{array}{l}\text { Look for proper arch support and know the life of the shoe } \\
\text { Allows for the patella to be manipulated into a certain position, allowing for } \\
\text { possible reduced friction } \\
\text { Apply for 10-20 min after activity }\end{array}$ \\
$\begin{array}{l}\text { Anti-inflammatories } \\
\text { Rest }\end{array}$ & $\begin{array}{l}\text { May help reduce symptoms during daily activities } \\
\text { Reduce (high impact) activity as this is an overuse injury }\end{array}$ \\
$\begin{array}{l}\text { Home exercise program } \\
\text { Use of low impact equipment (i.e. stationary bike, certain weight-lifting } \\
\text { programs) avoid high impact/loads to the knees }\end{array}$ & This can strengthen the quadriceps muscles \\
\hline
\end{tabular}




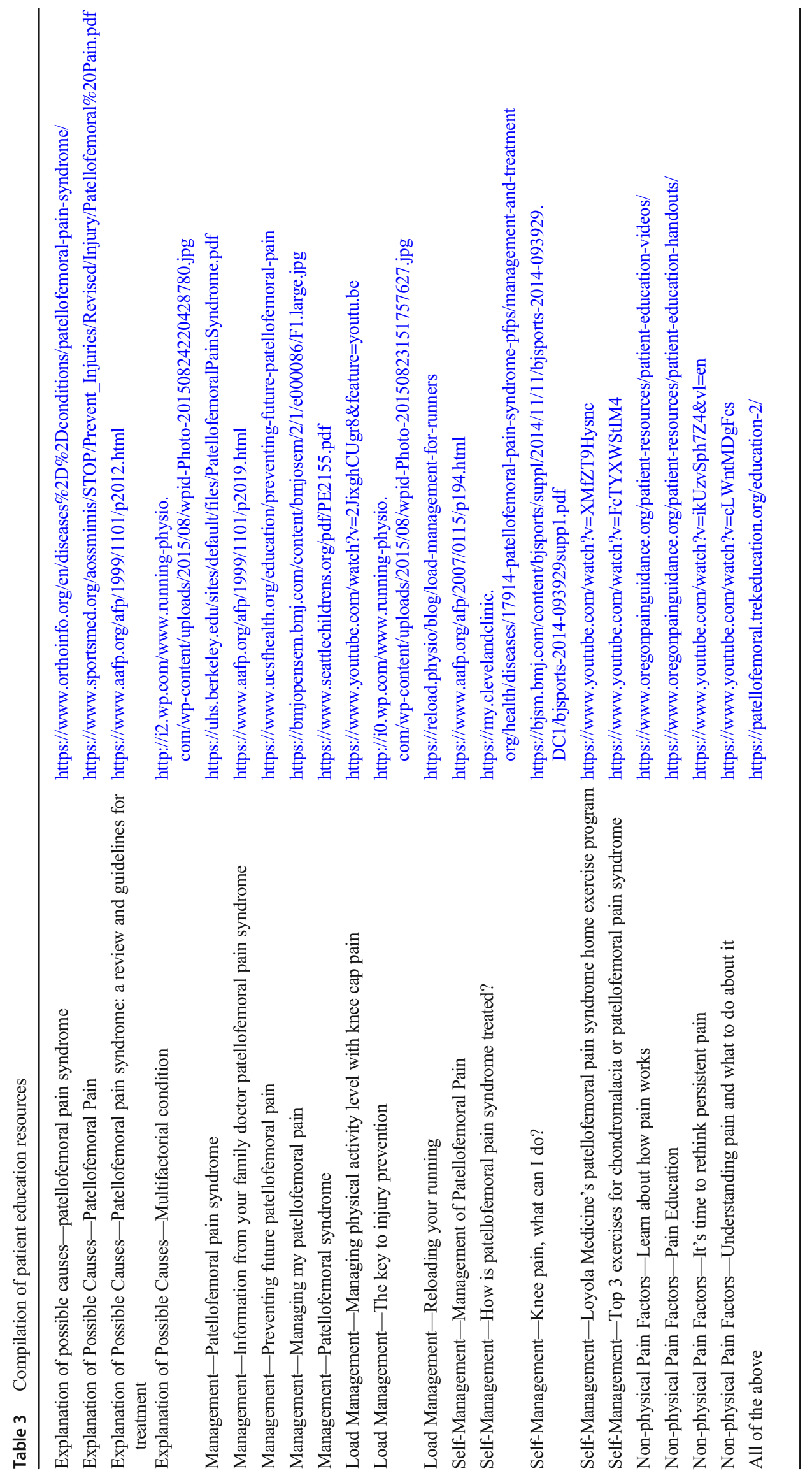




\section{Non-physical Factors}

Previously patellofemoral pain was thought to be related purely to local physical overloading of the bone, cartilage, and synovium [12]. However, patients with chronic patellofemoral pain demonstrate lower pain thresholds, widespread hyperalgesia, and impaired conditioned pain modulation, not only at the knee but also at distant sites throughout the body $[16,17]$. This finding suggests that there may be some additional central dysregulation component to chronic pain that would not be addressed by traditional treatment recommendations focusing only on the local physical factors. Patient education about these non-physical factors should therefore be included in a treatment plan for patients with these features.

Patients with patellofemoral pain have been found to have increased rates of other associated psychological factors: anxiety, depression, pain-related fear, fear avoidance, and catastrophizing thoughts. Furthermore, there are linear correlations between these psychological characteristics and measures of pain and physical function. Catastrophizing and painrelated fear demonstrated the strongest correlation with pain and function outcomes $[18 \bullet, 19]$. There is evidence to suggest that health care professionals with a strictly biomechanical explanation of pain are more likely to recommend that patients limit physical activity due to pain and, as a result, may foster fear avoidance and catastrophizing in their patients [20]. In patients with particularly high fear avoidance and catastrophizing thoughts, it may be helpful to introduce the idea that pain is not necessarily a result of tissue damage, but instead that some level of pain during physical activity is acceptable and not harmful [15•].

Other ways to address non-physical factors through patient education include assessing for unmet treatment expectations and eliciting patient engagement. Unmet treatment expectations have been identified as a barrier to treatment adherence. Understanding these expectations and helping set reasonable expectations are valuable ways to personalize the education that you provide. Eliciting patient engagement in the decisionmaking and treatment process is a facilitator to treatment adherence and promotes self-management [15॰].

Addressing non-physical factors may be one of the most challenging aspects of patient education, perhaps due to the stigma still surrounding anxiety, depression, and related conditions. An example of a good way to introduce this idea might be to make a broad normalizing statement like "research has shown that it is common for feelings of anxiety, depression or fear about pain to exacerbate this condition. Do you think that any of these feelings are coming up for you?" Although sometimes a difficult conversation to initiate, the simple act of introducing the idea of these non- physical factors will likely go a long way in developing patient insight into these contributing factors.

\section{Special Implications}

In the previously mentioned review by de Oliveira Silva et al., looking at the effectiveness of patient education, patient education was provided over an average of two visits. In comparison, when exercise-therapy was offered to the exercise group; an average of 20.5 visits was provided. Taping and gait retraining interventions likewise required more visits, with an average of 3.5 and 5 sessions respectively [6*0]. At the time of submission of this article, we are in the midst of a global pandemic, and over the past several months, healthcare systems have needed to restrict in-person visits and limit the number of visits to reduce transmission of COVID-19. These restrictions have highlighted the advantages of interventions like patient education, which require fewer visits and could help reduce transmission of communicable disease.

\section{Education Resources}

Traditionally, patient education has been provided verbally during a patient visit or handed to the patient in the written form of a handout or pamphlet. While these forms of education remain as good options for transmitting information, with more and more patients turning to the internet for their source of information, supplying patient education in the form of online or app-based is an attractive option. Through our own online searches and in conjunction with athletic training and physical therapy colleagues, we have attempted to compile a short list of examples within each category of content (Table 3) that may serve as resources or inspiration for individualized patient education for patellofemoral syndrome.

\section{Conclusion}

Although patient education was not mentioned specifically in the most recent international consensus statements as a recommended treatment for patellofemoral pain [3], these authors recommend dedicated patient education be included. Patient education alone may not be sufficient to optimize treatment outcomes in some cases, but adding patient education to other current treatments may increase the effectiveness or effective duration of those treatments, and is unlikely to cause any patient harm. Additionally, patient education is an attractive option in environments where limited contact with the patient is required. 


\section{Compliance with Ethical Standards}

Conflict of Interest Lauren Bosshardt and Taylor Ray declare that they have no conflict of interest.

Seth Sherman reports personal fees from Joint Restoration Foundation, personal fees from Smith and Nephew, personal fees from Vericel, personal fees from Arthrex, personal fees from ConMed, personal fees from Vivorte, personal fees from Zimmer, personal fees from Bioventus, personal fees from Epic Bio, outside the submitted work.

Human and Animal Rights and Informed Consent This article does not contain any studies with human or animal subjects performed by any of the authors.

\section{References}

Papers of particular interest, published recently, have been highlighted as:

- Of importance

•- Of major importance

1. Crossley KM, Stefanik JJ, Selfe J, Collins NJ, Davis IS, Powers CM, et al. 2016 Patellofemoral pain consensus statement from the 4th international patellofemoral pain research retreat, Manchester. Part 1: terminology, definitions, clinical examination, natural history, patellofemoral osteoarthritis and patient-reported outcome measures. Br J Sports Med. 2016;50:839-43.

2. Smith BE, Selfe J, Thacker D, Hendrick P, Bateman M, Moffatt F, et al. Incidence and prevalence of patellofemoral pain: a systematic review and meta-analysis. PLoS One. 2018;13:e0190892.

3. Collins NJ, Barton CJ, van Middelkoop M, Callaghan MJ, Rathleff MS, Vicenzino BT, et al. 2018 consensus statement on exercise therapy and physical interventions (orthoses, taping and manual therapy) to treat patellofemoral pain: recommendations from the 5th International Patellofemoral Pain Research Retreat, Gold Coast, Australia, 2017. Br J Sports Med. 2018;52:1170-8.

4. Collins NJ, Bierma-Zeinstra SMA, Crossley KM, van Linschoten RL, Vicenzino B, van Middelkoop M. Prognostic factors for patellofemoral pain: a multicentre observational analysis. Br J Sports Med. 2013;47:227-33.

5. Lankhorst NE, van Middelkoop M, Crossley KM, Bierma-Zeinstra SMA, Oei EHG, Vicenzino B, et al. Factors that predict a poor outcome 5-8 years after the diagnosis of patellofemoral pain: a multicentre observational analysis. Br J Sports Med. 2016;50: $881-6$.

6.• de Oliveira Silva D, Pazzinatto MF, Rathleff MS, Holden S, Bell E, Azevedo F, Barton C. Patient Education for Patellofemoral Pain: A Systematic Review. J Orthop Sports Phys Ther. 2020;50(7):388396. https://doi.org/10.2519/jospt.2020.9400. Very recently published systematic review suggesting that education interventions may produce similar outcomes in pain and function compared to exercise therapy plus education in patients with patellofemoral pain.

7. Bennell KL, Nelligan R, Dobson F, Rini C, Keefe F, Kasza J, et al. Effectiveness of an internet-delivered exercise and pain-coping skills training intervention for persons with chronic knee pain: a randomized trial. Ann Intern Med. 2017;166:453.

8. Bennell KL, Nelligan RK, Rini C, Keefe FJ, Kasza J, French S, Forbes A, Dobson F, Abbott JH, Dalwood A, Harris A, Vicenzino B, Hodges PW, Hinman RS. Effects of internet-based pain coping skills training before home exercise for individuals with hip osteoarthritis (HOPE trial): a randomised controlled trial. Pain. 2018;159(9): 1833-1842. https://doi.org/10.1097/j.pain.0000000000001281.

9. Coleman S, Briffa NK, Carroll G, Inderjeeth C, Cook N, McQuade $\mathrm{J}$. A randomised controlled trial of a self-management education program for osteoarthritis of the knee delivered by health care professionals. Arthritis Res Ther. 2012;14:R21.

10. Caldera F. Patellofemoral syndrome - a term to be avoided: response. Am J Sports Med. 2016;44:NP22.

11. Sisk D, Fredericson M. Update of risk factors, diagnosis, and management of patellofemoral pain. Curr Rev Musculoskelet Med. 2019;12:534-41.

12. Post WR, Dye SF. Patellofemoral pain: an enigma explained by homeostasis and common sense. Am J Orthop. 2017;46:92-100.

13. Nielsen RØ, Parner ET, Nohr EA, Sørensen H, Lind M, Rasmussen $\mathrm{S}$. Excessive progression in weekly running distance and risk of running-related injuries: an association which varies according to type of injury. J Orthop Sports Phys Ther. 2014;44:739-47.

14. Bandura A. Health promotion from the perspective of social cognitive theory. Psychol Health. 1998;13:623-49.

15. Smith BE, Moffatt F, Hendrick P, Bateman M, Selfe J, Rathleff MS, et al. Barriers and facilitators of loaded self-managed exercises and physical activity in people with patellofemoral pain: understanding the feasibility of delivering a multicentred randomised controlled trial, a UK qualitative study. BMJ Open. 2019;9: e023805 Highlights patient identified facilitators and barriers to effective education and implementation of self-management tools.

16. Noehren B, Shuping L, Jones A, Akers DA, Bush HM, Sluka KA. Somatosensory and biomechanical abnormalities in females with patellofemoral pain. Clin J Pain. 2016;32:915-9.

17. Holden S, Straszek CL, Rathleff MS, Petersen KK, Roos EM, Graven-Nielsen T. Young females with long-standing patellofemoral pain display impaired conditioned pain modulation, increased temporal summation of pain, and widespread hyperalgesia. PAIN. 2018;159:2530-7.

18. Maclachlan LR, Collins NJ, Matthews MLG, Hodges PW, Vicenzino B. The psychological features of patellofemoral pain: a systematic review. Br J Sports Med. 2017;51:732-42 Review article evaluating the correlations between psychological characteristics and patellofemoral pain severity.

19. Maclachlan LR, Collins NJ, Hodges PW, Vicenzino B. Psychological and pain profiles in persons with patellofemoral pain as the primary symptom. Eur J Pain. 2020. https://doi.org/10.1002/ ejp.1563.

20. Darlow B, Fullen BM, Dean S, Hurley DA, Baxter GD, Dowell A. The association between health care professional attitudes and beliefs and the attitudes and beliefs, clinical management, and outcomes of patients with low back pain: a systematic review. Eur J Pain. 2012;16:3-17.

Publisher's Note Springer Nature remains neutral with regard to jurisdictional claims in published maps and institutional affiliations. 